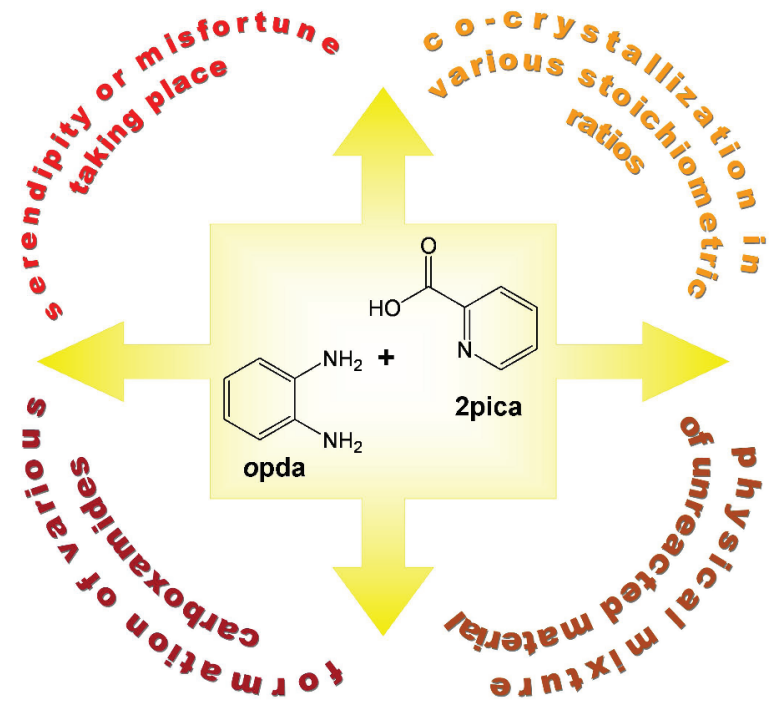

Figure 1. Schematic view of several possibilities of reaction of o-phenylenediamine (opda) and 2-picolinic acid (2pica).

Keywords: Carboxamide, Co-crystal, Solvothermal method, Solution based method, Grinding

\section{MS36-P5 Intermolecular interactions of benzimidazole derivatives}

Petra Bombicz ${ }^{1}$, Gyula Tamás Gál ${ }^{1}$, Ádám Lovász ${ }^{1}$, Nóra V. Nagy $^{1}$, Tamás Holczbauer ${ }^{1}$

1. Research Centre for Natural Sciences, Hungarian Academy of Sciences, POB 206, 1519 Budapest, Hungary

email: bombicz.petra@ttk.mta.hu

Imidazole is a constituent of the essential amino acid histidine what is present in many proteins and enzymes and plays a vital part in the structure and binding functions of hemoglobin. Imidazole is present in many pharmaceuticals, in antifungal, antiprotozoal and antihypertensive medications. It is a constituent of mercaptopurine, an immunosuppressive drug. There is benzimidazole moiety in vitamin $\mathrm{B}_{1}$. A number of substituted imidazoles are selective inhibitors of nitric oxide synthase, which makes drug targets in inflammation, neurodegenerative diseases and tumors of the nervous system. The thermostable polybenzimidazole contains imidazole fused to a benzene ring, and acts as a fire retardant. Imidazole has been used extensively as a corrosion inhibitor on certain transition metals, such as copper.

The way to the aim to produce new substances with required properties is based on the knowledge of the structural properties of widely characterised solids. There is a long time effort to influence or favourably fine tune structural properties of solid materials by substituents and / or guest molecules. Their different sizes, shapes and chemical composition consequently alter the physico-chemical properties. In a crystal both steric requirements and electrostatic forces play a role in the architecture. A given packing arrangement may tolerate small changes caused either by the gradual change in site and/or size of substitution or in guest molecules incorporated into a host lattice. When the tolerance is terminated a different packing arrangement and/or a different molecular conformation appears. Occasionally the packing motifs may still remain but the motifs are moved relative to each other. The non-covalent interactions have an influence on the packing arrangement and the molecular recognition processes.

We present on the example of a series of benzimidazole derivatives how the balanced spatial requirements and electrostatic forces play a role in the arrangement of packing motifs in the crystals. Influencing the intermolecular interactions shows how the supramolecular synthon can be engineered.

Keywords: intermolecular interactions, isomorphy, packing arrangement 